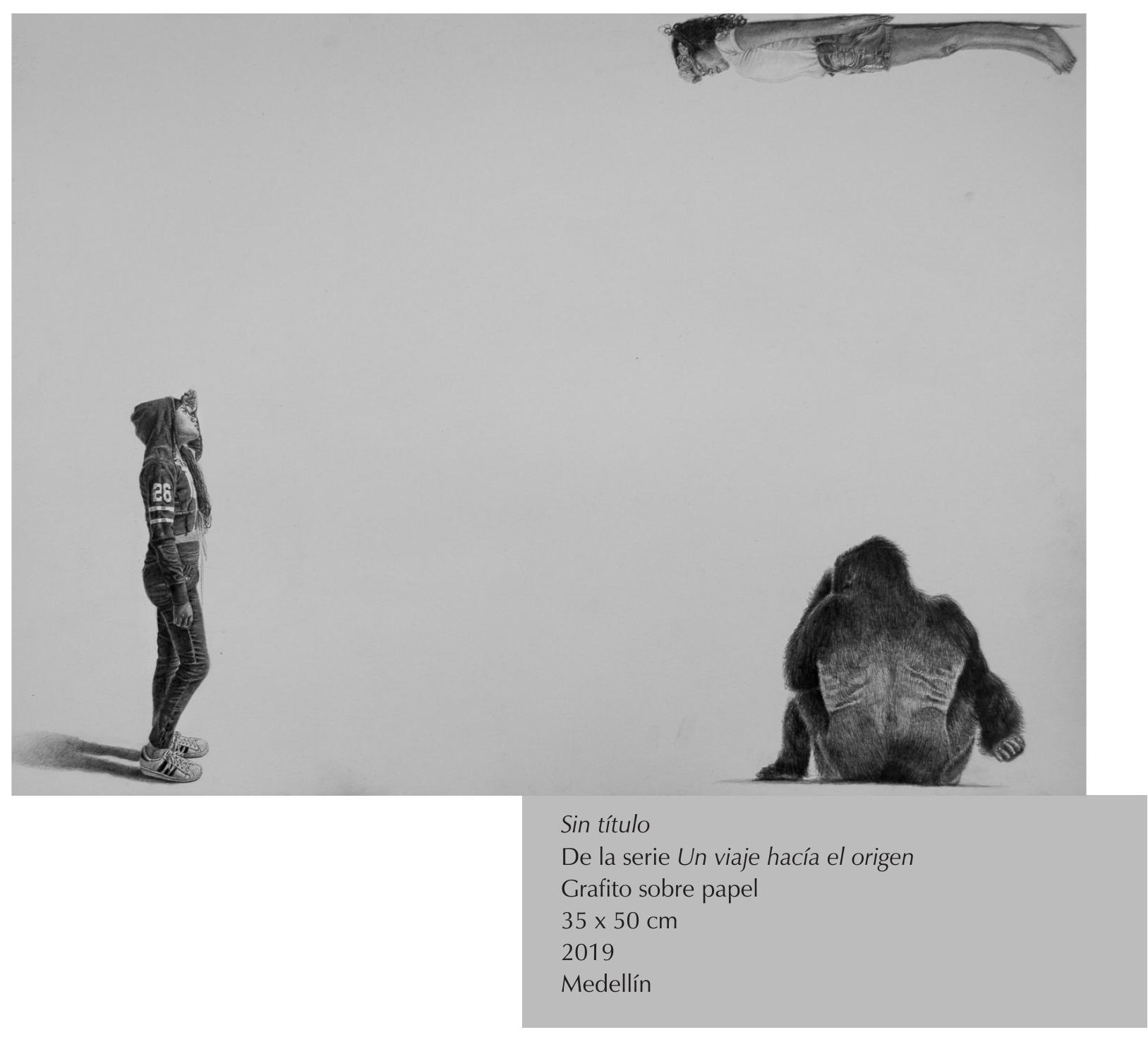




\title{
Configuración de subjetividades y violencia política en América Latina. Aportes a la emergencia y consolidación de un campo de estudios*
}

\author{
Martha Cecilia Herrera Cortés (Colombia)** \\ Vladimir Olaya Gualteros (Colombia)*** \\ Andrés Felipe Urrego Salas (Colombia) ${ }^{* * * *}$
}

\section{Resumen}

El artículo analiza la producción del programa de formación e investigación Configuraciones de Subjetividades y Constitución de Memorias sobre la Violencia Política en América Latina, con el propósito de identificar sus aportes en la emergencia y consolidación de un campo de estudios relacionado con esta problemática. Para ello se llevó a cabo un diseño bibliométrico descriptivo y retrospectivo, y la construcción de una red conceptual que permitió la revisión y análisis de la producción entre 2010 y 2017. De los resultados se destaca la adscripción del programa al enfoque de la historia reciente, los acercamientos interdisciplinarios para el tratamiento de los objetos de estudio, así como las articulaciones entre memoria, educación y formación, en contextos escolares y no escolares, para el estudio de las subjetividades.

\section{Palabras clave}

Violencia Política; Educación; Subjetividades; Memorias; América Latina; Colombia.

\footnotetext{
* Este artículo es producto del proyecto de investigación Programa de investigación y formación Configuración de subjetividades y constitución de memorias sobre la violencia política en América Latina: un estado del arte (código DPG-449-17), avalado y financiado por el Centro de Investigaciones, Universidad Pedagógica Nacional, Colombia.

** Socióloga. Magíster en Historia. Doctorada en Filosofía e Historia de la Educación. Profesora emérita y catedrática titular de la Universidad Pedagógica Nacional, Colombia, y directora del grupo de investigación Educación y Cultura Política de la misma universidad. Correo electrónico: malaquita10@gmail.com - Orcid: 0000-0003-4170-7822 - Google Scholar: https://scholar.google.es/ citations?hl=es\&user=ZbVZ7CcAAAAJ

*** Licenciado en Lingüística y Literatura. Magíster en Educación. Profesor ocasional y titular de la Universidad Pedagógica Nacional, Colombia, miembro del grupo de investigación Educación y Cultura Política de la misma universidad. Correo electrónico: vlado2380@gmail.com - Orcid: 00000002-3935-3271 - Google Scholar: https://scholar.google.es/citations?hl=es\&user=CSy6P8YAAAAJ

**** Licenciado en Filosofía. Miembro del grupo de investigación Educación y Cultura Política de la UniversidadPedagógica Nacional,Colombia.Correoelectrónico: afelipeurrego@gmail.com-Orcid:00000001-8408-3007 - Google Scholar: https://scholar.google.es/citations?hl=es\&user=0uiCbmcAAAAJ
} 
Fecha de recepción: agosto de 2018

- $\quad$ Fecha de aprobación: mayo de 2019

\title{
Cómo citar este artículo
}

Herrera Cortés, Martha Cecilia; Olaya Gualteros, Vladimir y Urrego Salas, Andrés Felipe. (2019). Configuración de subjetividades y violencia política en América Latina. Aportes a la emergencia y consolidación de un campo de estudios. Estudios Políticos (Universidad de Antioquia), 56, pp. 249-268. DOI: 10.17533/ udea.espo.n56a11

\section{Configuration of Subjectivities and Political Violence in Latin America. Contributions to the Emergence and Consolidation of a Field of Study}

\begin{abstract}
This article analyzes the creation of the program Configuration of Subjectivities and Conformation of Memories on Political Violence in Latin America (Configuraciones de Subjetividades y Constitución de Memorias sobre la Violencia Política en América Latina). It aims to identify its contributions to the emergence and consolidation of a field of study related to these issues. To this purpose, the study proposes a bibliometric descriptive and retrospective design, as well as the implementation of a social network that allowed for the revision and analysis of the production between 2010 and 2017. Among the results, we highlight the adscription of the program to the recent history approach, the interdisciplinary approaches for the treatment of the subjects of study, and the articulations between memory, education, and training in and out of school contexts for the study of subjectivities.
\end{abstract}

\section{Keywords}

Political Violence; Education; Subjectivities; Memories; Latin America; Colombia. 


\section{Introducción}

El campo de estudios en torno a la configuración de subjetividades en contextos de violencia política en América Latina se ha planteado como objetivo central - en contraste con estudios de corte macrosocialcomprender las experiencias de las víctimas, sus maneras de vivenciar $y$, si se quiere, de encarnar los fenómenos históricos vividos, así como visibilizar sus formas de enfrentar la violencia y los modos de subjetivación, desubjetivación y reconfiguración subjetiva en circunstancias asociadas a acontecimientos de violencia política (Jelin y Kaufman, 2006). En este orden, los trabajos de investigación buscan comprender, en términos amplios, las formas en que el fenómeno de la violencia política incide en la constitución de la experiencia de sí de los individuos.

Los quiebres dados en el tejido social y político, así como sus incidencias en las subjetividades a partir de los múltiples trastornos causados por la violencia, han Ilamado la atención de los gestores de las políticas públicas, de las organizaciones sociales, así como de los estudiosos de los temas sociales, políticos y culturales, generando diversas iniciativas en las que «las luchas por las memorias y por el sentido del pasado se convierten en un nuevo campo de la acción social en la región» (Jelin, 2003, p. 15). En este sentido, el campo de estudios se ha diversificado, revelando relaciones y luchas por una serie de capitales simbólicos y significados sociales que dicen, en buena medida, de la importancia de este tipo de saberes en el escenario de lo público (Bourdieu, 2002).

En países como Chile, Argentina, Uruguay, Perú, Colombia, entre otros, se llevan a cabo investigaciones con abordajes de diferentes disciplinas que se entrecruzan para estudiar la violencia política y su incidencia en las subjetividades. Se pueden identificar acercamientos lingüísticos, semióticos, literarios y estéticos que enfatizan en la importancia del lenguaje en la configuración de las subjetividades y en el procesamiento de la experiencia (Peris, 2008; Arfuch, 2002); psicosociales, cuyo eje de análisis son las incidencias de la violencia en las estructuras síquicas de los individuos, en el quiebre de los lazos sociales y en la violación a los derechos humanos (Lira y Loveman, 2005; Piper, 2015); sociológicos y politológicos, cuya preocupación se centra en la desestructuración del orden social y en los retos que tienen las sociedades en transición hacia la democracia para la recomposición del tejido social (Jelin, 2002; Lechner y 
Güell, 2006); antropológicos, de estudios culturales y de estudios visuales, enfocados en los nexos entre cultura e individuo, y sus dinámicas en torno al poder y a las significaciones culturales (Theidon, 2004; Cabrera, 2006; Castillejo, 2000; Degregori, 2004; Uribe-Alarcón, 2004); fenomenológicos, que procuran trazar los mapas existenciales de los sujetos, su mundo de vida, su discurrir cotidiano (D’abbraccio, 2007); históricos, que se detienen en el análisis de las políticas de memoria y el papel emergente del sujetovíctima en el marco de la historia reciente (Vezzetti, 2007; Crenzel, 2009; Hartog, 2012); y educativos y pedagógicos, interesados en los procesos formativos en contextos de violencia dentro y fuera de los circuitos escolares (Jelin y Lorenz, 2004; Rubio, 2013; De Amézola, 2008; Lizarralde, 2012; Rodríguez, 2014; Rodríguez y Sánchez, 2009; Arias, 2015; Herrera, Ortega, Cristancho y Olaya, 2013).

Todos estos acercamientos tienen en común la preocupación por la memoria, entendida como un artefacto cultural a partir del cual los seres humanos nos configuramos como sujetos en contextos históricos situados; en ellos se despliegan diversos usos de categorías como memoria individual, memoria social, memoria colectiva o memoria histórica (Stern, Winn, Lorenz y Marchesi, 2014). En su conjunto, estos acercamientos han contribuido al surgimiento de un campo de estudios en torno a los nexos entre memoria, violencia política y configuración de subjetividades, el cual es cada vez más pertinente para lograr la inteligibilidad de las dinámicas políticas y sociales contemporáneas en América Latina.

Entre las iniciativas surgidas en el contexto colombiano desde este tipo de acercamientos se encuentra el programa de formación e investigación Configuraciones de Subjetividades y Constitución de Memorias sobre la Violencia Política en América Latina, iniciado 2010 por el grupo de investigación Educación y Cultura Política de la Universidad Pedagógica Nacional (UPN). Este programa fue creado con el objetivo de propiciar investigaciones sobre las memorias en torno a acontecimientos de violencia política, para comprender su incidencia en la configuración de las subjetividades y en los procesos de formación ético-política. Desde esta perspectiva, el programa de formación e investigación se suma al campo de estudios antes mencionado, haciendo énfasis en los procesos de formación y ampliando la reflexión sobre lo educativo más allá de los problemas de enseñanza-aprendizaje, comunes a muchas investigaciones en educación. En otras palabras, el programa reflexiona sobre la forma en que los sujetos 
son «educados» por diversos acontecimientos sociales, en particular el conflicto armado y la violencia política. Es allí donde radica la importancia del programa, pues supera la idea de una comprensión de los fenómenos como ubicados en la línea del tiempo histórico, para reflexionar acerca de su incidencia en el tiempo presente y en los procesos de formación de los sujetos.

Es claro que quienes han agenciado este tipo de trabajos en la Universidad Pedagógica Nacional, si bien tienen un interés investigativo y académico, también los precede un propósito político: comprender la forma en que los fenómenos macrosociales —como la violencia políticainciden en los sujetos, al tiempo que se busca posicionar una perspectiva que sustrae lo educativo de un puro saber instrumental. Lo anterior significa pensar la educación y la formación como un lugar desde el que es posible ver y comprender los fenómenos sociales.

En esta perspectiva, la investigación sobre la que se basa el presente artículo llevó a cabo el análisis de la producción del programa a través de un estudio bibliométrico, complementado con un análisis de redes conceptuales, para identificar sus avances cualitativos y cuantitativos, las categorías que han orientado sus búsquedas y acercamientos epistemológicos, sus lugares de actuación y difusión, entre otros aspectos, con el fin de establecer los aportes del programa a la emergencia y consolidación de un campo de estudios sobre las relaciones entre violencia política y configuración de subjetividades en América Latina, así como su pertinencia para el ámbito educativo y de la formación política. De esta manera, en el artículo se presentan los principales hallazgos de la investigación, así como algunas conclusiones que se infieren a partir de ellos.

\section{Método}

\subsection{Diseño y unidad de análisis}

Se diseñó un estudio bibliométrico de tipo descriptivo y retrospectivo de la producción entre 2010 y 2017 de los ocho investigadores que integran el programa, incluyendo tanto productos publicados - artículos en revistas científicas y en publicaciones no indexadas, libros y capítulos de librocomo ponencias presentadas en eventos nacionales e internacionales. Para la selección de la unidad de análisis se procuró excluir los productos que hacían parte de investigaciones y temáticas ajenas al programa. Con base 
en ello se conformó un corpus documental con un total de 161 registros. Adicionalmente, se diseñó una red conceptual a partir de los artículos en publicaciones seriadas, tomando como referente las palabras clave que estos incluyen en su estructura.

\subsection{Procedimiento}

Para realizar las búsquedas se seleccionaron sitios web que contienen información sobre la producción de cada uno de los investigadores del programa y que permiten el acceso libre a los textos o brindan referencias sobre las revistas en las que se publica o las instituciones con las que se establecen relaciones. La ventana de observación de la información analizada fue de ocho años (2010-2017).

Se consultaron bases de datos como Redalyc, SciELO, Latindex y Publindex - en el caso de las revistas indexadas- y en las páginas web de las publicaciones periódicas no indexadas. Para complementar información sobre los artículos y encontrar registros sobre libros, capítulos de libro y ponencias se consultaron sitios como Google Scholar, Academia.edu y Researchgate, los repositorios de la Universidad Pedagógica Nacional y

[254] de las demás instituciones que los publicaron, así como los perfiles de los investigadores y del grupo Educación y Cultura Política en las plataformas CvLac y GrupLac de Colciencias.

Tras revisar que los documentos cumplieran con las características expuestas en el diseño metodológico se procedió a consolidar el corpus documental en el que se basó la investigación. Los diferentes registros se organizaron según indicadores bibliométricos como autor, título, año de publicación, revista o medio de publicación, palabras clave, tipo de colaboración, área temática y lugar de publicación. Para el análisis se llevó a cabo una sistematización en hojas de cálculo de Excel y su cuantificación en el software Statistical Package for the Social Sciences (SPSS). Para la construcción de la red conceptual se elaboró un gráfico precisando las redes de asociación entre las palabras clave de los artículos por medio de la herramienta de libre acceso NodeXL. También se consultaron las citaciones de artículos, libros y capítulos de libro a través de Google Scholar. Esto se complementó con fichas bibliográficas y analíticas para caracterizar cualitativamente las publicaciones. 


\section{Resultados}

Los investigadores que hacen parte del programa de formación e investigación provienen de disciplinas como filosofía, lingüística, historia, antropología, psicología, trabajo social, educación y pedagogía. La revisión de las trayectorias académicas de los integrantes del grupo evidencia su actuación prolongada en el campo educativo como maestros e investigadores, y con actividades de formación en escenarios comunitarios o no formales. La pertenencia y relación de los investigadores con campos de las ciencias sociales y humanas, así como los complejos contextos de violencia vivida en diferentes escenarios latinoamericanos, es quizás lo que posibilita y fortalece el interés por la formación más allá de lo institucional o lo restringido a las prácticas de enseñanza-aprendizaje. Los docentes del programa plantean una relación entre lo educativo y las ciencias sociales y las humanidades a través de sus trabajos de investigación, sus prácticas de formación y su producción académica. Este aspecto, en algún grado, da cuenta de la superación de la idea de que la educación tiene lugar en las ciencias sociales y las humanidades solo desde la aplicación o enseñanza de sus saberes. De esta manera, la producción académica del programa presenta un vínculo entre las diversas disciplinas que posibilita la construcción de conocimiento acerca de los fenómenos sociales. Como efecto de lo anterior, los investigadores participan en diferentes escenarios a través de sus publicaciones, con el interés de dar cuenta de hallazgos y una construcción de conocimiento que contribuya con la comprensión de nuestro pasado y presente conflictivos, pero también con el objetivo de participar, ser reconocidos y existir en la producción de conocimiento más allá de los tradicionales objetos de lo educativo.

En el sentido expuesto, las publicaciones tienen valor como evidencia de la construcción de conocimiento y como participación en un campo de saber. Así, se registra un total de 161 productos cuya autoría pertenece a los ocho investigadores adscritos al programa y a las colaboraciones que han establecido entre sí y con investigadores invitados o estudiantes vinculados en calidad de monitores, auxiliares o asistentes de investigación -el porcentaje de productos en colaboración con personas externas al equipo de investigadores es del $8,7 \%$, equivalente a 14 registros-.

De los 161 productos, 31 corresponden a artículos en revistas indexadas $(19,3 \%), 16$ a libros $(9,9 \%), 40$ a capítulos de libro $(24,8 \%), 59$ a ponencias 
presentadas en eventos nacionales e internacionales (36,6\%) y 15 a artículos en publicaciones no indexadas - como portales de opinión y revistas de difusión académica- $(9,3 \%)$. En las redes de coautoría predomina la tendencia a publicar en solitario $(n=116,72 \%)$, aunque también se registran publicaciones de dos $(n=31,19,3 \%)$, tres $(n=8,5 \%)$ y cuatro autores $(n=6$, $3,7 \%$ ). Hay un ritmo constante de producción anual y se registra mayor actividad entre 2012 y 2014.

Según las fichas bibliográficas y analíticas elaboradas, los registros se adscriben a disciplinas relacionadas con las ciencias sociales y las humanidades. Para esta distinción se plantea que las ciencias sociales son aquellas que estudian los fenómenos e instituciones sociales, por lo que en los productos se rastrean aportes de la pedagogía, la antropología, la ciencia política, la sociología y el trabajo social; mientras que las humanidades se relacionan con las estructuras y el entender propio de las expresiones humanas, por lo que allí encontramos soportes de disciplinas como los estudios visuales, el cine, la lingüística, la literatura, la filosofía y la historia. Para el estudio bibliométrico se clasificaron y cuantificaron, aparte de la producción del área de ciencias sociales, la correspondiente a educación y pedagogía, ya que esta es el área en la que se adscribe el programa estudiado, motivo por el cual se consideró pertinente mostrar esta producción especificada.

En cuanto a los 31 artículos en revistas indexadas, hay publicaciones colombianas $(n=23,74,2 \%)$, así como de otros países latinoamericanos $(n=5,16,1 \%)$ y europeos $(n=2,9,4 \%)$. De acuerdo con las clasificaciones temáticas registradas en Latindex y Publindex, 15 de estos artículos se encuentran en revistas de educación y pedagogía (48,4\%), 15 en revistas de otras disciplinas de las ciencias sociales y humanas $(48,4 \%)$ y uno en una revista multidisciplinar $(3,2 \%)$.

Como se hace evidente, la producción académica, resultado del programa de investigación y formación, demuestra un descentramiento de los temas particulares de la enseñanza. En este sentido se puede suponer que el programa ha aceptado, apropiado y resignificado una serie de apuestas teóricas y epistemológicas de las ciencias humanas y sociales. En otras palabras, se asumen categorías, se reconocen unos trayectos históricos y unas formas de percepción que coadyuvan a ver la educación y la formación en una dimensión amplia. 
En la tabla 1 se presentan las revistas indexadas en las que se ha publicado en relación con su país de origen y el porcentaje de artículos que se registra en cada una.

Tabla 1. Revistas indexadas donde se registran artículos.

\begin{tabular}{|l|l|c|}
\hline \multicolumn{1}{|c|}{ Nombre de la revista } & País de origen & $\begin{array}{c}\text { Porcentaje } \\
\text { de artículos } \\
\text { en la revista }\end{array}$ \\
\hline Análisis Político & Colombia & $3,2 \%$ \\
\hline Cinémas d'Amérique Latine & Francia & $3,2 \%$ \\
\hline Ciudad Paz-Ando & Colombia & $3,2 \%$ \\
\hline Cuadernos de Filosofía Latinoamericana & Colombia & $3,2 \%$ \\
\hline Cuadernos de Música, Artes Visuales y Artes Escénicas & Colombia & $6,5 \%$ \\
\hline Educação e Filosofia Uberlândia & Brasil & $3,2 \%$ \\
\hline Espacios en Blanco & Argentina & $3,2 \%$ \\
\hline Historia Crítica & Colombia & $3,2 \%$ \\
\hline Historia de la Educación. Anuario & Argentina & $3,2 \%$ \\
\hline $\begin{array}{l}\text { Imagofagia. Revista de la Asociación Argentina de Estudios } \\
\text { de Cine y Audiovisual }\end{array}$ & Argentina & $3,2 \%$ \\
\hline Kamchatka. Revista de Análisis Cultural & & $3,5 \%$ \\
\hline Nodos y Nudos & España & $3,2 \%$ \\
\hline Nómadas & Colombia & $6,5 \%$ \\
\hline Pedagogía y Saberes & Colombia & $12,9 \%$ \\
\hline Pro-Posições & Colombia & $3,2 \%$ \\
\hline Prospectiva. Revista de Trabajo Social e Intervención Social & Colombia & $3,2 \%$ \\
\hline Revista Colombiana de Educación & Colombia & $19,4 \%$ \\
\hline Revista de Estudios Sociales & Colombia & $3,2 \%$ \\
\hline Revista Virtual Universidad Católica Del Norte & Colombia & $3,2 \%$ \\
\hline Voces y Silencios: Revista Latinoamericana de Educación & Colombia & $3,2 \%$ \\
\hline & & \\
\hline
\end{tabular}

Fuente: elaboración propia.

Asimismo, en cuanto a los 15 registros de publicaciones seriadas no indexadas estos se encuentran en sitios colombianos, 2 aparecen en la revista de difusión académica Educación y Cultura (13,3\%) y 13 en portales de opinión sobre temas políticos y de actualidad como Palabras al Margen $(n=10,66,7 \%)$, El Espectador $(n=2,13,3 \%)$ y Las Dos Orillas $(n=1,6,7 \%)$. 
Los artículos presentan una diversidad temática reflejada en las áreas bajo las que se clasifican las revistas; no obstante, hay categorías de análisis que son transversales a los estudios y dan cuenta de un horizonte interpretativo común del que se apropian los investigadores según el objeto de estudio al que se acercan y el área disciplinar en la que se sitúen, lo cual va decantando una red conceptual que da sentido al quehacer investigativo. Para rastrear dichas categorías se construyó una red conceptual con base en las palabras clave de los artículos que arrojó un total de 145 palabras entre las que se distinguieron 61 diferentes (gráfica 1).

Los nodos que denotan más entrecruzamientos parten de las categorías de memoria, violencia política, subjetividades, formación y cultura política. Es posible inferir que con esto se entreteje una red de inteligibilidad en la que las subjetividades en contextos de violencia política se comprenden bajo el lente de procesos de formación y del lugar que ocupa la memoria como dispositivo para su configuración. A la luz de estas relaciones, se hace uso de la categoría de cultura política para indicar las articulaciones entre las dinámicas políticas y las culturales, así como los entrelazamientos dados entre lo individual y lo social. En esta línea, emerge el concepto de ecologías violentas para denominar a los contextos de violencia política y dar cuenta de la serie de elementos que inciden de manera simultánea en su conformación y en sus dinámicas, afectando las culturas políticas.

El análisis de la red también denota la importancia de la pedagogía de la memoria, que se vincula con la categoría de historia reciente, las cuales se despliegan en trabajos en los que se indaga sobre las políticas de la memoria y su expresión en los lineamientos y propuestas de enseñanza en torno al pasado reciente, por ello también se entrecruzan con la formación, la memoria y la violencia política. La historia reciente da cuenta de un enfoque historiográfico que, desde acercamientos interdisciplinares a los objetos de estudio, reivindica las memorias de los sujetos, pues se centra en las diferentes voces y huellas que dan cuenta de los efectos del pasado en el presente, al tiempo que se interpelan las memorias hegemónicas al posicionar en la esfera pública a sujetos excluidos de la historia oficial.

También se encuentran registros que abordan análisis sobre literatura testimonial elaborada por personas que experimentaron directamente regímenes autoritarios en América Latina, así como trabajos en artes visuales que presentan versiones e interpretaciones de lo sucedido desde el cine, el 
Configuración de subjetividades y violencia política en América Latina...




documental, el arte callejero, la fotografía, las narrativas ficcionales, entre otros formatos. En este sentido, el programa ha dado importancia al análisis de registros relacionados con las expresiones estético-artísticas, las cuales develan aproximaciones novedosas a las relaciones entre arte, estética y política para pensar la configuración de las subjetividades.

Respecto a los eventos en donde se han presentado ponencias, estos convocan investigadores en educación, humanidades y ciencias sociales, a partir de temas como las narrativas, las políticas públicas, el conflicto armado y la memoria histórica, así como hay eventos menos específicos sobre trabajos en disciplinas como la pedagogía, la filosofía, el trabajo social y la historia. La mayoría de estos eventos han tenido lugar en Colombia $(n=44$, $74,6 \%)$, también hay registros en varios países latinoamericanos $(n=10$, $16,9 \%)$, europeos $(n=4,6,8 \%)$ y en Estados Unidos $(n=1,1,7 \%)$. Asimismo, puede verse que de los $161,36,6 \%$ lo ocupa este tipo de producción, lo cual es indicativo de un gran dinamismo en este ámbito académico, lo que permite la socialización entre pares de los avances investigativos, así como el posicionamiento de la producción del programa.

En general, en términos conceptuales, en algunos trabajos se habla [260] de ecologías violentas para caracterizar aquellas espacialidades en las que, por la mediación de la violencia política, rige una lógica de la eliminación física y simbólica del otro para imponer modelos identitarios individuales y colectivos que inciden en la configuración de las subjetividades. La producción estudiada destaca que la subjetividad se configura a partir de las experiencias vitales de los sujetos en su relación con otros y consigo mismo, señalando, al mismo tiempo, los estrechos nexos entre procesos de formación y constitución de subjetividades; a su vez, la formación se ha entendido como el conjunto de procesos por los cuales los sujetos se apropian de su situación histórico-cultural para comprenderse, para asumir posiciones y valores, y para reflexionar sobre sus prácticas y transformarlas. Dichos conceptos se han abordado en concordancia con el de cultura política, el cual se ha comprendido como el entramado de significados y acciones que se construye en torno a las relaciones de poder en espaciotemporalidades determinadas; asimismo, la memoria se describe como un rasgo constituyente de las subjetividades que no se comprende como homogénea, sino que conlleva distintos modos de recordar de acuerdo con la diversidad de sujetos que pueden dar cuenta de ella, por lo que es posible hablar de resistencias y luchas por el reconocimiento que conllevan procesos 
de reconfiguración subjetiva y restitución de los lazos sociales fragmentados que se dan en escenarios diversos.

En este sentido, han sido importantes las reflexiones en torno a los usos políticos de la memoria, pues permiten visibilizar las pendularidades entre el sujeto que perfilan los discursos estatales y el sujeto que exige sus derechos y reivindica sus memorias con denuncias y críticas a los discursos y mandatos oficiales. Así, en algunas de las publicaciones se muestra la emergencia de grupos y movimientos sociales que ejercen este tipo de resistencias desde diferentes ámbitos: militantes de movimientos políticos que han hecho oposición a los regímenes de derecha en el continente; mujeres y sus agenciamientos a partir de las opresiones que viven por su condición de género; propuestas artísticas y culturales de jóvenes, estudiantes, sectores populares y organizaciones de maestros, entre otros.

En cuanto a las publicaciones que han citado la producción académica del programa de formación e investigación, la mayoría son artículos científicos, tesis y trabajos de grado. Hay una tendencia de publicaciones sobre la enseñanza del pasado reciente y de la violencia política en relación con la formación de maestros, la educación en derechos humanos y las pedagogías de la alteridad y de la memoria, por lo que las citas aparecen en publicaciones de disciplinas como la pedagogía, la historia y la antropología, las cuales plantean propuestas didácticas y problemas referidos a la enseñanza tanto en escenarios formales como no formales. De igual modo, son recurrentes las tesis y artículos sobre los modos de tramitar los traumas de la violencia política en América Latina desde ámbitos como el psicosocial y el artístico, que proponen estrategias para reconstruir los lazos sociales y las trayectorias de vida que se vieron afectadas por los conflictos violentos del continente en las últimas décadas; en este sentido, se registran publicaciones de disciplinas como la psicología, los estudios culturales y los estudios artísticos que presentan formas de analizar y enfrentar los fenómenos violentos a partir de diversas acciones de movimientos artísticos y culturales que surgen en torno a ello. En un sentido similar se registran publicaciones de áreas como la teología y la salud pública, en las que se abordan propuestas para enfrentar las consecuencias del conflicto armado desde las dimensiones médicas y espirituales de los sujetos que han vivido este tipo de situaciones; además, se evidencia repercusión en artículos sobre las relaciones de los medios de comunicación y el conflicto armado, las expresiones artísticas y culturales en torno a la memoria, los estudios sobre la infancia y la juventud en contextos 
de violencia política y las reflexiones acerca de conceptos relacionados con las víctimas, la ciudadanía y la formación de los sujetos. Este tipo de temas se encuentran en documentos de disciplinas como la sociología, la filosofía, la ciencia política y la comunicación social. Las publicaciones en donde se registran citas de la producción del programa son de países como Colombia, Argentina, España, México, Brasil y Bolivia.

\section{Discusión}

En términos generales, la producción investigativa del programa muestra el uso que se hace para el tratamiento de los objetos de estudio de diversas herramientas teóricas y metodológicas procedentes de las ciencias sociales y humanas, lo cual revela un tipo de hacer investigativo que se ubica más en la interdisciplinariedad que en una disciplina específica, lo cual coincide con una de las características de la historia reciente, a la que se adscribe el programa. Esta perspectiva interdisciplinar no se asume en tanto se reúnan diferentes disciplinas para analizar un mismo fenómeno, sino en la medida en que el estudio de la configuración de subjetividades en contextos de violencia política es un campo interrelacional en el que se articulan diferentes aspectos de lo social y lo humano, es decir, los mismos

[262] objetos de estudio lo exigen.

Lo anterior incide de manera favorable en la receptividad que encuentra la producción del programa en los espacios de mayor posicionamiento en estas áreas, incluyendo colaboraciones editoriales, revistas, eventos e interacciones entre pares, lo cual potencia la capacidad de incidencia del conocimiento producido por el equipo de trabajo. Este posicionamiento se percibe en que de los 31 artículos en revistas científicas indexadas $51,6 \%$ está en publicaciones multidisciplinarias o propias de las áreas de las ciencias sociales y humanas. Asimismo, es pertinente resaltar que $48,4 \%$ de los artículos se encuentra en revistas que se adscriben al área de la educación y la pedagogía, lo cual es explicable en la medida en que el programa funciona en una universidad formadora de docentes, cuya mayor preocupación es impactar las prácticas docentes y los procesos de formación pedagógica. Además, por el tipo de publicaciones en donde se cita la producción del programa, así como por las temáticas y disciplinas a las que estas pertenecen, se puede inferir que hay repercusión en diferentes escenarios académicos en donde se genera conocimiento en el marco del campo de estudios sobre la configuración de subjetividades en contextos 
de violencia política en América Latina, estos escenarios dan cuenta de las intersecciones disciplinares que se han dado para comprender y tramitar las diferentes coyunturas políticas en el continente.

Como bien sabemos, publicar en revistas indexadas es uno de los mayores retos de los investigadores debido a sus altos requerimientos, motivo por el cual aparecer en ellas es señal de reconocimiento de la producción del grupo y de la aceptación de unas normas y un tipo de razonamientos, al tiempo que significa la posibilidad de obtener mayor circulación de la producción gracias a las bases de datos nacionales e internacionales que sistematizan y regulan los estándares de calidad de este tipo de publicaciones. En la actualidad, con sus pros y contras, la idoneidad de los investigadores se valora no solo por su capacidad investigativa, sino también por lograr formatos comunicativos que permitan una difusión amplia del conocimiento alcanzado, por lo que al mismo tiempo se valora por su posicionamiento en las revistas científicas indexadas ya que ellas se consideran uno de los canales de difusión académica más importantes (Schriewer, 2006).

Si bien, es de destacar la amplia presencia de la producción analizada en revistas científicas indexadas, es preciso visibilizar también las publicaciones en libros y capítulos de libro - que representan $34,7 \%$ de la producción-, toda vez que este vehículo ha sido uno de los predilectos de los investigadores que trabajan en las ciencias sociales y humanas, y aunque este no tiene las posibilidades de circulación que tienen las revistas indexadas, permite la exposición detallada de los resultados investigativos o de las discusiones en torno a las categorías de análisis que dinamizan un campo de estudios.

En cuanto al trabajo que ha caracterizado las actividades del grupo, es posible decir que este ha dado lugar a aportes epistemológicos y metodológicos desde los que se ha producido teoría. De esta manera, se han realizado investigaciones sobre acontecimientos de violencia política referentes a la historia reciente de Argentina, Chile y Colombia en las que se rastrea su incidencia en la configuración de subjetividades, pretendiéndose que la experiencia de estos países ilumine la colombiana, sin aludir propiamente a estudios comparados pero sí a historias conectadas que, habidas sus propias especificidades históricas, encuentran puntos en común que pueden ser examinados desde una perspectiva relacional. Desde allí se ha contribuido a mostrar cómo se configuran subjetividades, tanto 
desde las interpelaciones dadas por los poderes hegemónicos como desde la resistencia a los mismos, visibilizando sus expresiones en el ámbito cultural y político.

Las formas de ver y comprender el mundo social en medio de ecologías violentas se dinamizan por efecto de actores, situaciones e incluso discursos que afectan tanto las subjetividades como la construcción de significaciones sociales y que se expresan en un orden de la memoria, marcando, a su vez, pautas para las actuaciones de los sujetos en la esfera pública. Por lo anterior, posibilitar procesos de formación ético-política en medio de contextos de violencia política exige pensar en los modos como colectivos e individuos se han configurado a la luz de sus prácticas y de sus repertorios de memoria, con el fin de posibilitar procesos de reconfiguración subjetiva a partir de la valoración de la vida, la dignidad, el respeto a los derechos y a la diferencia.

\section{Conclusiones}

La historia reciente de América Latina ha estado marcada por acontecimientos de violencia política que han incidido de manera notable

[264] en el tejido social e institucional, así como en la configuración de las subjetividades de quienes fueron víctimas directas o simplementevivenciaron, en cuanto ciudadanos, alguno de los regímenes políticos dictatoriales o represivos que caracterizaron el continente a partir de la década de 1970. El interés por comprender esta problemática ha dado pie a diversas iniciativas dentro de las que se cuentan propuestas formativas e investigativas que han posibilitado la constitución de un campo de estudios interesado en la configuración de las subjetividades en contextos de violencia política en la historia reciente latinoamericana, como es el caso del programa de formación e investigación Configuraciones de subjetividades y constitución de memorias sobre la violencia política en América Latina.

El análisis de la producción y sus aportes, Ilevado a cabo a través del estudio bibliométrico y de la red conceptual, permitió rastrear una labor de largo aliento de casi una década. Desde allí se ha producido teoría y soportes epistémicos y metodológicos sobre los temas de estudio desde una perspectiva interdisciplinar. Se puede decir que hay una producción constante y canales de difusión reflejados en las revistas en las que se publica, 
la cantidad de eventos en los que se presentan ponencias y los lugares donde se desarrollan, así como los libros y capítulos de libros publicados y los lugares en donde se referencian estos productos.

El análisis de la producción académica y de sus redes conceptuales nos permite hablar de una suerte de hibridación, en donde se resignifican y apropian los métodos e instrumentos de varias disciplinas para comprender y analizar un objeto de estudio particular, lo que ayuda a configurar o fortalecer un campo de estudios. Sumado a ello, dicha hibridación, evidente en la producción intelectual y en las prácticas formativas en torno al análisis de los procesos de formación de subjetividades al interior de ecologías violentas, produce no solo un movimiento en los conocimientos de los investigadores, sino que repercute en las significaciones de las disciplinas en las que estos se movilizan. A su vez, la producción académica del programa dinamiza el campo de estudios en torno a la configuración de subjetividades en contextos de violencia política, en tanto devela nuevas relaciones; quizás la de mayor significación tiene que ver con la posibilidad de ver los procesos de formación y educación ligados a configuraciones sociales y construcciones éticas y políticas, las que se expresan en narrativas y en diversas expresiones culturales. En otras palabras, dicha hibridación no solo permite ver la forma de encarnar una serie de fenómenos sociales, sino los modos en que se transforma la experiencia de sí en la constitución de diferentes posiciones y momentos en las trayectorias formativas de los sujetos.

Pese a las dinámicas presentadas, se hace necesario incrementar los indicadores de colaboración tanto entre el equipo de investigadores como con redes y grupos de trabajo afines a esta problemática. En este mismo sentido, se requiere aumentar el porcentaje de publicaciones en revistas indexadas internacionales, lograr mayor presencia en eventos internacionales y profundizar en el estudio de experiencias latinoamericanas diferentes a Colombia. Todo ello en aras de potenciar el trabajo y obtener mayor interacción con los investigadores que contribuyen con sus conocimientos al fortalecimiento de este campo de estudios.

A partir de los resultados de la investigación, puede verse que el programa ha mostrado con sus trabajos la pertinencia de emprender procesos formativos en espacios escolares y no escolares que posibiliten 
la construcción de culturas políticas cuyos repertorios ayuden a configurar sujetos críticos, conscientes del pasado y con capacidad de agenciamiento en la esfera política. Esto ha dado lugar a investigaciones sobre pedagogía de la memoria y formación ético-política, por lo general acompañadas de propuestas de enseñanza y herramientas didácticas, planteadas desde los contextos de los grupos y movimientos sociales que han sido reprimidos y en diálogo con sus luchas.

En esta dirección, el programa ha contribuido desde sus diferentes iniciativas al posicionamiento de un campo de estudios que se ha estado conformando en América Latina desde décadas atrás, a partir de las reflexiones generadas en distintos países del continente con el fin de entender y tramitar los repertorios que la violencia política ha decantado en torno a nuestra historia reciente, para poder así reconocer su incidencia en la configuración de las subjetividades contemporáneas e identificar los retos que en este ámbito existen en relación con la formación política.

\section{Referencias bibliográficas}

1. Arfuch, Leonor. (2002). El espacio biográfico. Dilemas de la subjetividad [266] contemporánea, Buenos Aires: Fondo de Cultura Económica.

2. Arias Gómez, Diego Hernán. (2015). La enseñanza de la historia reciente y la formación moral. Dilemas de un vínculo imprescindible. Folios, 42, pp. 29-41. DOI: 10.17227/01234870.42folios29.41

3. Bourdieu, Pierre. (2002). Campo de poder, campo intelectual. Itinerario de un concepto. Tucumán: Montressor.

4. Cabrera, Martha. (2006). Exceso y defecto de la memoria: violencia política, terror, visibilidad e invisibilidad. Oasis, 11, pp. 39-55.

5. Castillejo, Alejandro. (2000). Poética de lo otro. Antropología de la guerra, la soledad y el exilio interno en Colombia. Bogotá, D. C.: Ministerio de Cultura, Icanh.

6. Crenzel, Emilio. (2009). Los derechos humanos y las políticas de la memoria. Reflexiones a partir de las experiencias de las comisiones de la verdad de Argentina y Chile. En: Vinyes, Ricard (ed.). El Estado y la memoria. Gobiernos y ciudadanos frente a los traumas de la historia (pp. 357-367). Buenos Aires: Del Nuevo Extremo.

7. D’abbraccio Kreutzer, Guillermo. (2007). Al borde de la cornisa: Construcción cotidiana del mundo social en el conflicto armado colombiano. (Tesis inédita de doctorado). Flacso, México, D. F.

8. De Amézola, Gonzalo. (2008). Esquizohistoria. La historia que se enseña en la escuela, la que preocupa a los historiadores y una renovación posible de la historia escolar. Buenos Aires: Libros del Zorzal. 
9. Degregori, Carlos. (2004). Heridas abiertas, derechos esquivos: reflexiones sobre la Comisión de la Verdad y Reconciliación. En: Belay, Raynald; Bracamonte, Jorge; Degregori, Carlos y Vacher, Jean Joinville (eds.). Memorias en conflicto. Aspectos de la violencia política contemporánea (pp.75-85). Lima: Embajada de Francia en el Perú, IEP, IFEA, Red para el Desarrollo de las Ciencias Sociales en el Perú. DOI: 10.4000/books.ifea.562

10. Hartog, Francois. (2012). El tiempo de las víctimas. Revista de Estudios Sociales, 44, pp.12-19. DOI: 10.7440/res44.2012.02

11. Herrera, Martha Cecilia; Ortega Valencia, Piedad; Cristancho, José Gabriel y Olaya, Vladimir. (2013). Memoria y formación: configuraciones de la subjetividad en ecologías violentas. Bogotá, D. C.: Universidad Pedagógica Nacional. DOI: 10.2307/j. ctvfc52rg

12. Jelin, Elizabeth. (2002). Los trabajos de la memoria. Madrid: Siglo XxI.

13. Jelin, Elizabeth. (2003). Los derechos humanos y la memoria de la violencia política y la represión: la construcción de un campo nuevo en las ciencias sociales. Cuadernos del IDES, 2. Recuperado de http://biblioteca.clacso.edu.ar/gsdl/collect/ar/ ar-025/index/assoc/D4331.dir/cuaderno2_Jelin.pdf

14. Jelin, Elizabeth y Kaufman, Susana (comps.) (2006). Subjetividad y figuras de la memoria, Buenos Aires: Siglo xxı.

15. Jelin, Elizabeth, Lorenz, Federico. (2004). Educación y memoria: la escuela elabora el pasado. Madrid: Siglo xxı.

16. Lechner, Norbert y Güell, Pedro. (2006). Construcción social de las memorias

en la transición chilena. En: Jelin, Elizabeth y Kaufman, Susana (comps.). Subjetividad y figuras de la memoria. Buenos Aires: Siglo xxı.

17. Lira, Elizabeth y Loveman, Brian. (2005). Políticas de reparación: Chile 19902004. Santiago: LOM, Universidad Alberto Hurtado, Dibam.

18. Lizarralde, Mauricio. (2012). La escuela y la guerra, las memorias entre el miedo y el silencio. Praxis Pedagógica, 3, pp. 90-103. DOI: 10.26620/uniminuto. praxis.12.13.2012.90-103

19. Microsoft Excel. (2007-2016). NodeXL: Network Overview, Discovery and Exploration for Excel. [Software de cómputo]. Recuperado de https://archive.codeplex. $\mathrm{com} / ? \mathrm{p}=$ nodexl

20. Peris, Jaume. (2008). Historia del testimonio chileno. De las estrategias de denuncia a las políticas de la memoria. Revista Quaderns de Filologia, 21, (anejo N. ${ }^{\circ}$ LXIV). Recuperado de http://roderic.uv.es/handle/10550/25163

21. Piper, Isabel. (2015). Violencia política, miedo y amenaza en lugares de memoria. Athenea Digital, 15 (4), pp. 155-172.

22. Rodríguez, Sandra y Sánchez, Marlene. (2009). Problemáticas de la enseñanza de la historia reciente en Colombia: Trabajar con la memoria en un país en guerra. Reseñas de Enseñanza de la Historia, 7, pp. 15-66. 
23. Rodríguez, Sandra. (2014). Enseñanza y aprendizaje de la historia en Colombia, 1990-2011. En: Plá, Sebastián y Pagès, Joan (coords.). La investigación en enseñanza de la historia en América Latina (pp. 109-154). México, D. F.: Bonilla Artigas, Universidad Pedagógica Nacional.

24. Rubio, Graciela. (2013). Memoria, política y pedagogía. Santiago: LOM.

25. Schriewer, Jürgen. (2006). Internacionalidades múltiples: surgimiento de una ideología a nivel mundial y persistencia de criterios idiosincráticos mundiales. En: Charle, Christophe; Schriewer, Jürgen y Wagner, Peter (comps.). Redes intelectuales trasnacionales. Formas de conocimiento académico y búsqueda de identidades culturales (pp. 359-408). Barcelona-México, D. F.: Pomares.

26. Stern, Steve; Winn, Peter; Lorenz, Federico y Marchesi, Aldo (eds.). (2014). No hay mañana sin ayer. Batallas por la memoria histórica en el Cono Sur. Santiago: Lom.

27. Theidon, Kimberly. (2004). El conflicto armado interno y la política de la reconciliación en el Perú. Lima: IEP.

28. Uribe Alarcón, María Victoria. (2004). Antropología de la Humanidad. Un ensayo interpretativo sobre el terror en Colombia. Bogotá, D. C.: Norma.

29. Vezzetti, Hugo. (2007). Conflictos de la memoria en la Argentina. Un estudio histórico de la memoria social. Historizar el Pasado Vivo en América Latina. Recuperado de http://www.historizarelpasadovivo.cl/es_resultado_textos.php?cate goria $=$ Argentina $\% 3 \mathrm{~A}+\mathrm{el}+$ tiempo + largo $+\mathrm{de}+\mathrm{la}+$ violencia + pol\%EDtica\&titulo [268] = Conflictos + de + la + memoria + en + la + Argentina. + Un + estudio + hist\%F3rico + $\mathrm{de}+\mathrm{la}+$ memoria + social 